\title{
SURVIVAL AND TREATMENT OUTCOMES OF PEDIATRIC SOFT TISSUE AND BONE SARCOMAS IN TANZANIA
}

\section{Zephania Saitabau Abraham ( $\sim$ zsaitabau@yahoo.com )}

The University of Dodoma College of Health Sciences https://orcid.org/0000-0002-7738-9902

\section{Siwillis Elizabeth Mithe}

Kenya Ministry of Health

Nazima Dharsee

Muhimbili University of Health and Allied Sciences

Trish Scanlan

Muhimbili University of Health and Allied Sciences

Mamsau Ngoma

Muhimbili University of Health and Allied Sciences

Josephine Kahiu

University College London Medical School

Lynn Million

Stanford University

\section{Research article}

Keywords: Soft tissue sarcoma (STS), Rhabdomyosarcoma (RMS), Non-rhabdomyosarcoma soft tissue sarcoma (NRSTS), Bone Sarcoma, Osteosarcoma, Ewing sarcoma, Treatment, Treatment Outcome, Overall Survival, Muhimbili, Tanzania.

Posted Date: July 29th, 2019

DOI: https://doi.org/10.21203/rs.2.12086/v1

License: (c) (1) This work is licensed under a Creative Commons Attribution 4.0 International License. Read Full License 


\section{Abstract}

Background Pediatric sarcomas represent an important group of childhood tumors who require treatment at Muhimbili National Hospital (MNH), the largest pediatric oncology center in Tanzania. Treatment is often adapted from established childhood protocols validated in clinical trials from the United States and United Kingdom. There are no studies describing the outcomes of treatment in Tanzania to understand similarities and disparities with other countries. The objective of this study was to describe the treatment outcomes including the overall survival for children seen at $\mathrm{MNH}$. Methods Data on treatment outcome was collected on all children seen at MNH pediatric oncology unit between 2011 and 2016 with a confirmed histological diagnosis of either bone or soft tissue sarcoma. Results A total of 135 cases were analyzed; 89 (66\%) were Soft tissue sarcoma (STS) and 46 (34\%) were bone sarcomas. In the STS group, 37 (41.6\%) achieved a complete response, 12 (13.5\%) achieved a partial response and 40 (44.9\%) had no response. Factors found to be significantly associated with a higher complete response rate included early stage disease (stage I or II), embryonal histology, tumor in favorable sites and tumor size of $<5 \mathrm{~cm}$ for STS and $<8 \mathrm{~cm}$ for the bone sarcomas. There was a trend for better 2-year overall survival for STS in early stage disease $(74.5 \%)$ compared to late stage disease $(20.6 \%), p<0.001$. A similar trend was noted for the bone sarcomas; $91.7 \%$ versus $25.3 \%$ for early stage and late stage disease respectively, $p=0.001$. Conclusions This report is the first study documenting the treatment outcome for pediatric sarcomas seen in Tanzania. Disease stage is strongly related to treatment outcome with later stages of the disease having an overall poor prognosis.

\section{Background}

The mainstay of treatment for both pediatric bone and soft tissue sarcomas is surgery. Radiation therapy (RT) and chemotherapy (chemo) may be added in selected cases depending on grade and other factors.

For localized soft tissue sarcomas, wide excision surgery is the standard treatment for all patients. This is done upfront if possible. In general, adjuvant radiation therapy is considered after wide excision for highgrade, deep $>5 \mathrm{~cm}$ lesions with close or positive microscopic margins, but only for the subgroup of tumors that are known to be radiation sensitive. RT is administered post-operatively at doses ranging from 40-60 gray (Gy) at 1.8-2Gy per fraction [1]. In some non-resectable tumors or borderline resectable tumors, available options include induction chemotherapy with or without RT and reassessment for surgical resection. For patients with advanced metastatic disease, chemotherapy is the standard treatment in Europe and North America [1].

For bone sarcomas, surgery with or without chemotherapy is the mainstay of treatment with the aim of surgery being to preferably preserve limb function as much as possible without compromising disease control [2]. Most current protocols include a period of preoperative chemotherapy, although this has not been proven to add survival benefit compared to postoperative chemotherapy alone [2]. 
In Tanzania, management is guided through treatment protocols adapted from clinical trials run from Children's Oncology Group (COG) and the International Society of Pediatric Oncology (SIOP) with therapy modified based on drug availability, surgical and radiation therapy resources. Currently, there is no treatment outcome data for pediatric sarcomas in Tanzania. The objective of this study was to establish the outcomes of treatment including overall survival of children with bone and soft tissue sarcomas in Tanzania.

\section{Methods}

\section{Study design and setting}

This was a descriptive retrospective review of pediatric patients aged between 0 to 18 years with a histopathologically confirmed diagnosis of either a soft tissue or bone sarcoma at Muhimbili National Hospital Pediatric Oncology unit from January 2011 to December 2016.

\section{Study participants}

Patients aged between 0 to 18 years were recruited in this study

\section{Data collection tool}

Data extraction forms were used to retrieve data from patients' records stored in manual files and or files on the computer and it captured type of treatment modalities and outcome of treatment.

\section{Sample size}

A total of 135 paediatric patients with histopathologically confirmed diagnosis of either a soft tissue or bone sarcoma

\section{Data processing and analysis}

The statistical analysis was done using the SPSS version 20.0 for windows. Continuous variables were summarized by means, medians, standard deviation and range. Categorical variables were summarized by frequencies, percentages and bar graphs for general description. Kaplan Meier curves were used to identify and compare overall survival. As one of the assumptions of using the log rank test, outcomes consisted of two mutually exclusive and collective exhaustive states; 'censored' and 'events'. 'Events' was the outcome of interest, in this case death. 'Censored' included outcomes such as alive and/or lost to follow up. A probability value of $<0.05$ was considered statistically significant.

The outcome variables were:

- Complete response (CR) -Disappearance of all target lesions.

- Partial response (PR) -At least a one third (30\%) decrease in the sum of the largest diameter (LD) of target lesions. 
- No response (NR) -Shrinkage of measurable disease but one which does not achieve a one third $(30 \%)$ or greater reduction which is insufficient tumor shrinkage to qualify as PR.

- Overall survival -Interval between diagnosis and death from any cause.

Recurrence -Disease that has come back usually after a period of time during which the cancer could not be detected

\section{Ethical considerations}

Ethical clearance was obtained from the Muhimbili University of Health and allied sciences (MUHAS) Research Ethics and Publication Committee prior to implementation of this study. A waiver of informed consent was also requested and granted by the same committee and permission to conduct the study was obtained from $\mathrm{MNH}$ as per hospital management protocols.

\section{Results}

A total of 135 cases of histologically confirmed either bone or soft tissue sarcoma fulfilled the criteria and were included in the final analysis; 89 (66\%) of the cases were STS and $46(34 \%)$ were bone sarcomas. Table 1 shows the modality of treatment received by the eligible patients. Surgery was the most commonly applied modality of treatment across both soft tissue and bone sarcomas followed by chemotherapy.

Table 1 shows modality of treatment given to the patients. Surgery $82(60.7 \%)$ was the most commonly applied modality of treatment followed by chemotherapy $74(54.8 \%)$. The palliation group included those patients who were palliated upfront with best supportive care only $26(19.3 \%)$.

Figure 1 shows the type of surgery done (either curative or palliative) in these patients. Wide local excision (WLE) was the most common surgical method used for the STS patients ( $41 \%$ for RMS and $52 \%$ for the NRSTS). WLE for the bone cases included procedures such as hemi-mandibulectomy. About one half of bone sarcoma cases were treated with curative surgery (amputation) and the other half were not treated with any surgical method but were rather given best supportive care. About $15 \%$ of the patients received palliative surgery (debulking/salvage).

Table 2 shows the type of chemotherapy regimen prescribed for these patients. MAP (30\%) was the most commonly prescribed regimen in the bone sarcoma category while IVA $(62.1 \%)$ was the most common regimen among the STS although about half of the patients not given any chemo but rather received best supportive care. 
Table 3 shows treatment response in relation to tumor size. In the STS category, patients with tumor size of $>5 \mathrm{~cm}$ had higher no response rates(43.1\%) compared to patients with tumor size of $<5 \mathrm{~cm}$ who had higher complete response rates(47.4\%).

In the bone sarcoma category, the same trend was noted, patients with tumor size of $>8 \mathrm{~cm}$ had higher no response rates $(75 \%)$ compared to those with tumor size of $<8 \mathrm{~cm}$ who had higher complete response rates $(50 \%)$.

Table 4 shows treatment response in relation to disease site. For the STS category and with regard to the H\&N site, $40.5 \%$ of the patients with NPM sites involvement had a CR while only $5.4 \%$ of the cases with $\mathrm{PM}$ involvement had a $\mathrm{CR}$. With regard to the $\mathrm{GU}$ site, only $2.5 \%$ of the patients with $\mathrm{B} / \mathrm{P}$ involvement had a CR whereas $8.1 \%$ of patients with NB/P involvement had CR. For the bone sarcomas category, $20 \%$ of the patients with $\mathrm{H} \& \mathrm{~N}$ disease had a CR while $80 \%$ of patients with disease at the extremities had a CR; $75 \%$ of the cases with disease at the extremities had a NR and $21.4 \%$ of the head and neck cases had NR.

Table 5 shows treatment response in relation to disease stage. Children who presented with stage I disease had $100 \%$ complete response (CR) rate while those who presented with late stage disease had higher no response (NR) rates in both STS and bone sarcomas. In the STS category, $100 \%$ of children with stage IV embryonal and alveolar RMS had NR. In the bone sarcomas category, $88.9 \%$ of patients with stage IV osteosarcoma had no response (NR) while 100\% of the Ewing's cases in the same category had NR.

Figure 2 shows that the 3 year and 5-year overall survival rates for the STS were $38.4 \%$ and $26.4 \%$ respectively. The mean survival time was 29.5 months, $95 \%$ confidence interval (22-37) and median survival time was 15 months, $95 \%$ confidence interval (8.9-21.1).

Figure 3 shows that the survival distribution for the different soft tissue sarcoma (STS) disease stages was significantly different $x^{2}=24.29, p<0.001$. Early stages (stage I and II) had better overall survival rates than the late stages (stage III and IV).

Figure 4 shows that the survival distribution for the different rhabdomyosarcoma (RMS) histological subtypes was significantly different $x^{2}=7.56, p=0.006$. The embryonal RMS had better overall survival 
rates compared to its alveolar counterpart.

Figure 5 shows that the 3 -year overall survival rate for the bone sarcomas was $37.5 \%$. The mean survival time was 23.4 months, $95 \%$ confidence interval (16.9-29.9) and median survival time was 13.6 months, $95 \%$ confidence interval (1.4-25.8).

Figure 6 shows the overall survival for bone sarcomas in relation to the disease stage. The survival distribution for all the different bone sarcoma stages was significantly different $x^{2}=11.7, p=0.001$. Early stages (stage I and II) had better overall survival rates than the late stages (stage III and IV).

\section{Discussion}

Treatment for the RMS cases is based on the risk stratification. The alveolar RMS is usually categorized as high risk irrespective of the disease stage or the post op clinical group. In this study, $29 \%$ of the embryonal RMS cases had high risk disease, $6 \%$ had intermediate risk while $40 \%$ had low risk disease.

At the MNH pediatric oncology unit, the EpSSG protocol is used in children with non-metastatic RMS. According to this protocol, children with low risk RMS are treated with the two drug combination VA regimen given in a total of 8 blocks (22 weeks). Children with intermediate (standard) risk RMS are treated with the VAC/IVA regimen(s) in a total of 9 blocks ( 25 weeks) if they had either PR or CR after the first 3 blocks. Patients with high risk RMS are treated with either IVA or IVADo (Ifosfamide, Vincristine, Actinomycin D and Doxorubicin) regimens. Those with locally advanced NRSTS are treated with surgery and chemotherapy (depending on histology) whiles those with advanced stage NRSTS are palliated upfront. The EURAMOS protocol (MAP regimen) is employed for children with osteosarcoma requiring chemotherapy while those with advanced stage disease are palliated upfront. Patients with Ewing's sarcoma are treated with the EURO-WING 99 protocol (VIDE regimen) followed by surgery and postoperative chemotherapy (IVA/VAC) if they have positive resection margins.

In this study, low risk RMS patients were initially treated using the IVA regimen (in 9 blocks) until August 2016 when the protocol was changed to the VAC regimen (change of the alkylating agent from Ifosfamide to Cyclophosphamide for dosage and toxicity reasons). The VAC regimen is also used in Europe and America for low risk patients [3]. In India however, a 22 week VA (Vincristine and Actinomycin D) regimen is considered effective for low risk RMS patients [4].

Children with high risk RMS in India and America are treated with VI chemotherapy followed by RT from week 13 or concurrent VI or VC chemotherapy plus RT from week 1 for those requiring emergency RT from week $1[3,4]$. In this study, patients with high risk RMS (like the alveolar RMS) were palliated upfront as these tumors have shown to frequently relapse or progress despite initial chemotherapy and are 
associated with an overall poor prognosis (although previously they were treated with IVA or IVADo chemotherapy regimens). The NRSTS were treated using either IVA or VAC regimens in this study.

A significant percentage of children with osteosarcoma in this study had stage IV disease at presentation $(47.8 \%)$ and were palliated upfront. The remaining percentage of osteosarcoma cases who presented with early stage disease were treated with pre and post-operative chemotherapy using a modified version of the EURAMOS protocol; the MAP regimen (omitting high dose methotrexate). Europe and America use a similar protocol [5]. The Ewing's sarcomas were treated with the VIDE regimen in this study.

In the STS category in this study, patients with smaller tumor size (less than $5 \mathrm{~cm}$ ) had higher rates of complete response (47.4\%) compared to those with larger tumor size (more than $5 \mathrm{~cm}$ ) who had higher rates of no response $(43.1 \%)$ and the difference was statistically significant, $p=0.03$. Size was reported to be an important treatment outcome determinant in STS studies done in India and Korea $[4,6]$. In the bone sarcoma category, the same trend was noted; patients with smaller tumor size (less than $8 \mathrm{~cm}$ ) had higher complete response rates $(50 \%)$ compared to those with a larger tumor size (more than $8 \mathrm{~cm}$ ) who had a higher no response rate $(75 \%)$ and the difference was borderline significant, $p=0.05$.

With regard to site in this study, in the STS category, patients with non PM sites involvement had better response to treatment (CR) compared to those with PM sites involvement (40.5\% vs. $5.4 \%)$ respectively. Patients with GU non B/P sites involvement had better $\mathrm{CR}$ rates compared to those with B/P sites involvement ( $8.1 \%$ vs. $2.5 \%$ ) respectively but these differences were not statistically significant, $p=0.7$. In the bone sarcomas category, patients with disease at the extremities had a higher complete response rate $(80 \%)$ compared to those with head and neck disease $(20 \%)$ though again the difference was not statistically significant, $p=0.5$.

In this study, the 3 year and 5-year overall survival rates for the STS were $38.4 \%$ and $26.4 \%$ while the 3 year and 5-year overall survival rates for RMS in particular were $39.2 \%$ and $36.8 \%$ respectively. This varies slightly with overall survival data from other studies. In the SEER data for RMS, the 5-year overall survival rate was about 64\% [7]. According to Lim Sun Min et al in a study done in Korea the 5 year overall survival was $45 \%$ for RMS(6) while it was also $45 \%$ in the South African study by Aziza V.D.S et al [9] and $58.7 \%$ according to the study by Hendricks et al [8]. The 2-year overall survival estimates in relation to the disease stage were as follows for the STS category: early stages of the disease (stage I and II) had better 2-year overall survival rates (74.5\%) than the late stages (stage III and IV) $20.6 \%, p<0.001$. According to the South African study by Hendricks M. et al for RMS only the 5-year survival rates by stage were as follows: stage I (80\%), stage II (70\%), stage III (54.1\%), and stage IV (38.5\%) [8]; generally showing that early disease stages had better overall survival rates compared to the late disease stages. In another South African study on RMS by Aziza V.D.S et al, $55 \%$ of the survivors had stage III disease while only $13 \%$ had stage IV disease. Early stages of the disease had survival rates of up to $70 \%$ [9]. The 3-year OS for the bone sarcomas was $37.5 \%$ in this study. The 5 -year overall survival for osteosarcoma in the study done in Netherlands was $66.9 \%$ [10]. Similarly, for bone sarcomas early stages of the disease (stage I and 
II) had better 2-year overall survival rates (91.7\%) compared to the late stages (stage III and IV) $25.3 \%$, $p=0.001$.

With respect to histology for the RMS in this study, patients with embryonal RMS had better 2-year overall survival rates compared to those with alveolar RMS, $57 \%$ vs. $6.4 \%(p=0.006)$. This could be explained by the fact that alveolar RMS is known to frequently relapse and even progress despite initial chemotherapy. For bone sarcomas and in relation to histology, the Ewing's sarcoma surprisingly had better 2-year overall survival rates compared to the osteosarcomas (60\% vs. $40 \%)$ but the difference was not statistically significant $(p=0.8)$. This non-statistically significant difference may be explained by the significantly lower number of Ewing's sarcoma cases demonstrated in this series.

\section{Conclusions}

Site, size, stage and histology of these sarcomas are important outcome determinants.

\section{Abbreviations}

COG: Children's Oncology Group MNH: Muhimbili National Hospital; NRSTS: Non-rhabdomyosarcoma soft tissue sarcoma; SIOP: International Society of Pediatric Oncology; STS: Soft tissue sarcoma; RT: Radiotherapy; RMS: Rhabdomyosarcoma;

\section{Declarations}

Ethical approval: Ethical clearance was obtained from the Senate Research and Publications Committee of Muhimbili University of Health and Allied Sciences. Permission to conduct the study was obtained from $\mathrm{MNH}$ authority as per hospital management protocols.

Consent for publication: Not applicable

Availability of data and materials: All relevant data pertinent to this research can be obtained from the corresponding author upon a reasonable request.

Competing interests: The authors declare that they have no competing interests

Funding: None

Authors' contributions: ZSA, SEM, ND, TS, MN, JK and LM participated in the conception and designing of the study, collection, analysis and interpretation of data. All authors prepared the manuscript and approved the final draft of such manuscript.

Acknowledgements: Special appreciation goes to MNH for provision of a conducive environment for conducting our study. 


\section{References}

1. Clinical E, Guidelines P. Soft tissue and visceral sarcomas: ESMO clinical practice guidelines for diagnosis, treatment andfollow-up. Ann Oncol. 2012;23(SUPPL. 7). 2. Hogendoorn PCW, Athanasou N, Bielack S, De Alava E, Dei Tos AP, Ferrari S, et al. Bone sarcomas: ESMO Clinical Practice Guidelines for diagnosis, treatment and follow-up. Ann Oncol Off J Eur Soc Med Oncol. 2010;21 Suppl 5(Supplement 5):v204-13. 3. Walterhouse DO, Pappo AS, Meza JL, Breneman JC, Hayes-Jordan AA, Parham DM, et al. Shorter-Duration Therapy Using Vincristine, Dactinomycin, and Lower-Dose Cyclophosphamide With or Without Radiotherapy for Patients With Newly Diagnosed Low-Risk Rhabdomyosarcoma: A Report From the Soft Tissue Sarcoma Committee of the Children's Oncology . J Clin Oncol [Internet]. 2014 Nov 1;32(31):3547-52. Available from: http://www.ncbi.nlm.nih.gov/pmc/articles/PMC4209105. 4. Kapoor G, Das K. Soft tissue sarcomas in children. Indian J Pediatr. 2012;79(7):936-42. 5. Whelan JS, Bielack SS, Marina N, Smeland S, Jovic G, Hook JM, et al. EURAMOS-1, an international randomised study for osteosarcoma: Results from pre-randomisation treatment. Ann Oncol. 2015;26(2):407-14. 6. Lim SM, Yoo CJ, Han JW, Cho YJ, Kim SH, Ahn JB, et al. Incidence and Survival of Pediatric Soft Tissue Sarcomas: Comparison between Adults and Children. Cancer Res Treat [Internet]. 2015 Jan 21;47(1):9-17. Available from: https://doi.org/10.4143/crt.2013.157 7. Ries L a. G, Smith M a., Gurney JG, Linet M, Tamra T, Young $\mathrm{JL}$, et al. Cancer incidence and survival among children and adolescents: United States SEER Program 1975-1995. NIH Pub No 99-4649. 1999;179 pp. 8. Hendricks M, Parkes J, Pillay K, Millar A, van Eyssen A, Numanoglu A, et al. Outcomes of children with rhabdomyosarcoma treated with intensive chemotherapy, surgery, and radiotherapy through a period of protocol revision at a South African center, 1990-2010. Pediatr Blood Cancer [Internet]. 2017;64(3):n/a-n/a. Available from: http://dx.doi.org/10.1002/pbc.26248 9. Van Der Schyff A, Stefan DC. Clinical characteristics and outcome of rhabdomyosarcoma in South African children. African J Haematol Oncol. 2010; 10. Hagleitner MM, De Bont ESJM, Te Loo DMWM. Survival trends and long-term toxicity in pediatric patients with osteosarcoma. Sarcoma. 2012;

\section{Tables}

Due to technical limitations, tables are only available as a download in the supplemental files section.

\section{Figures}


Figure 1: Type of Surgery done

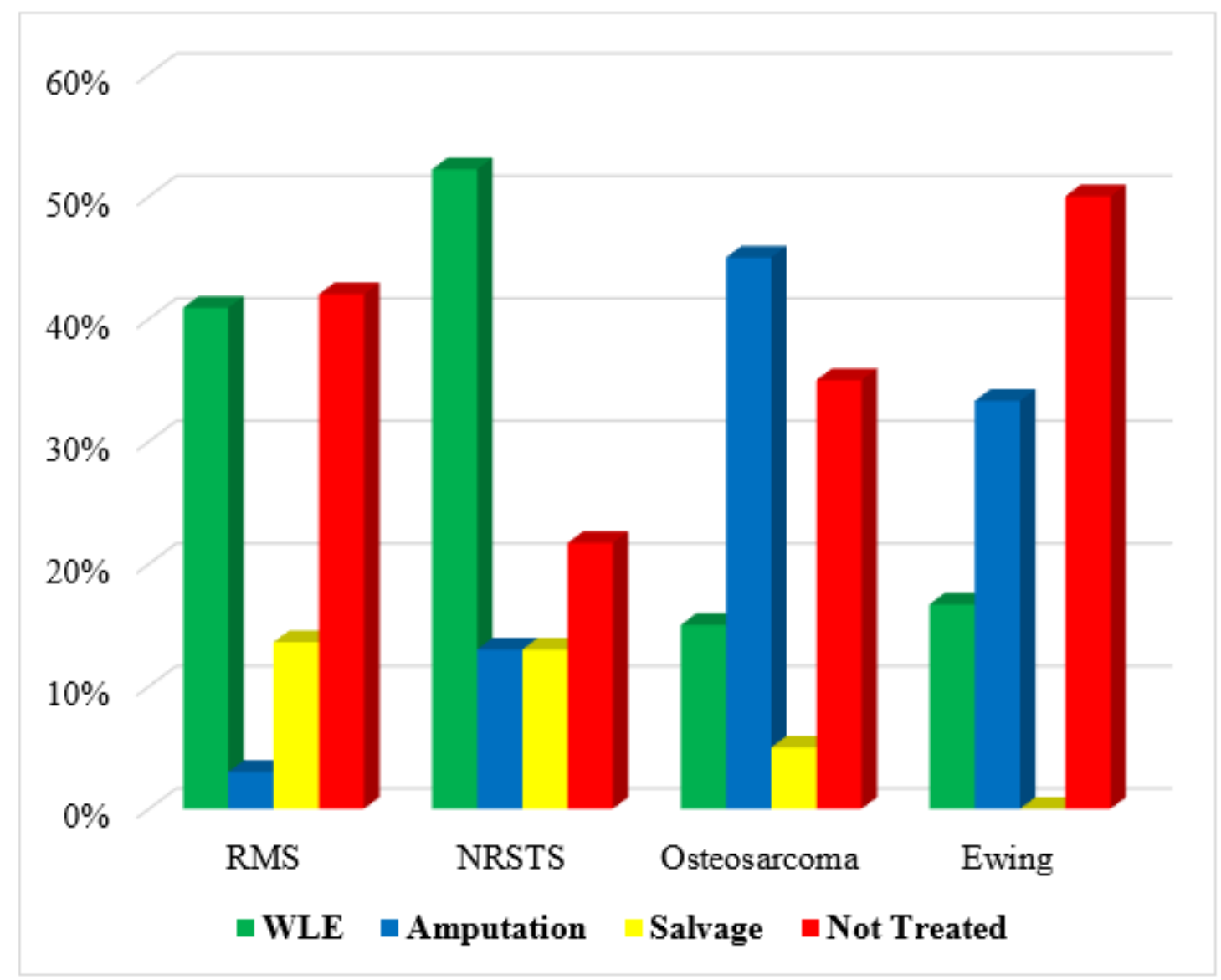

Abbreviations: WLE, Wide Local Excision; RMS, rhabdomyosarcoma; NRSTS, non rhabdomyosarcoma soft tissue sarcomas.

Figure 1

type of surgery done 
Figure 2: Overall Survival rate of STS

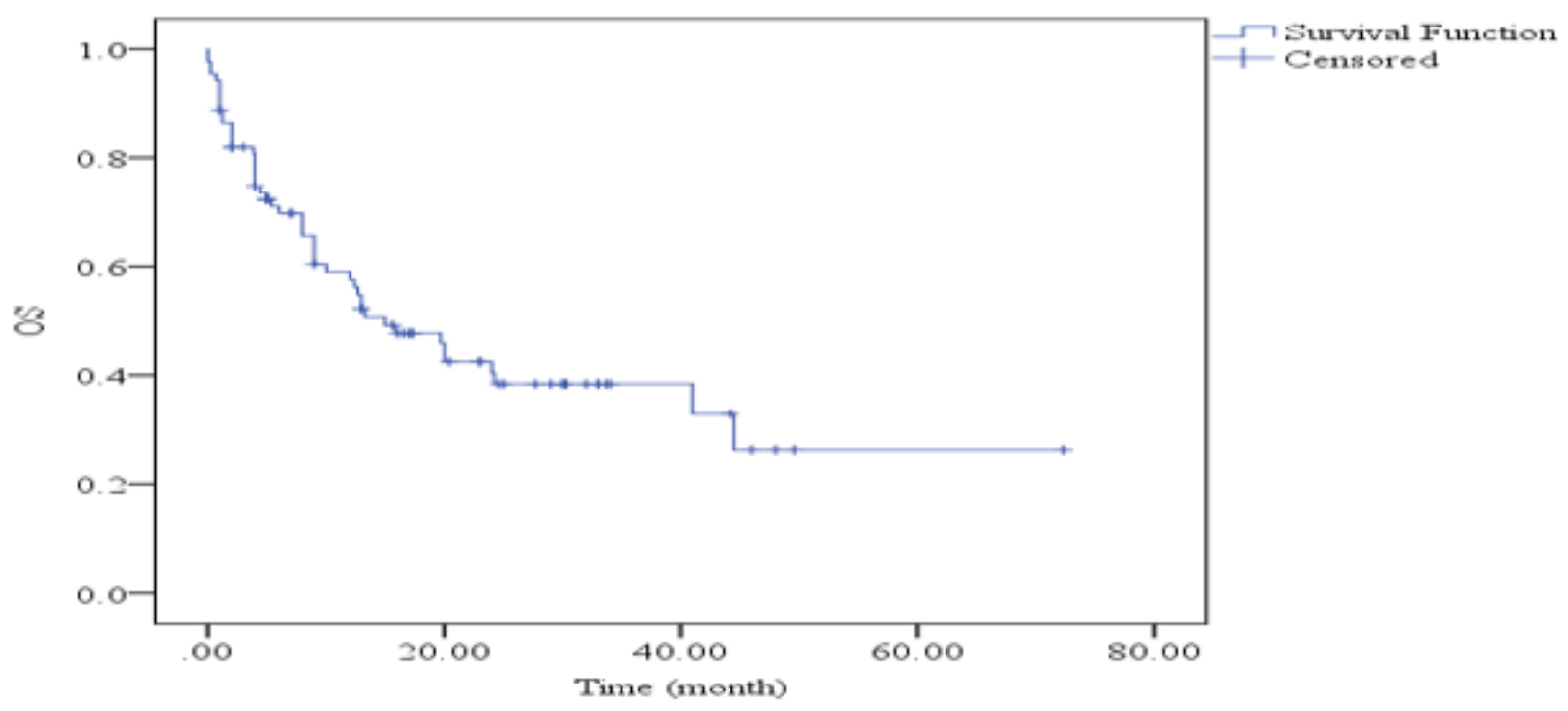

Figure 2

overall survival rate of STS

Figure 3: Overall Survival estimates of STS in relation to the disease stage

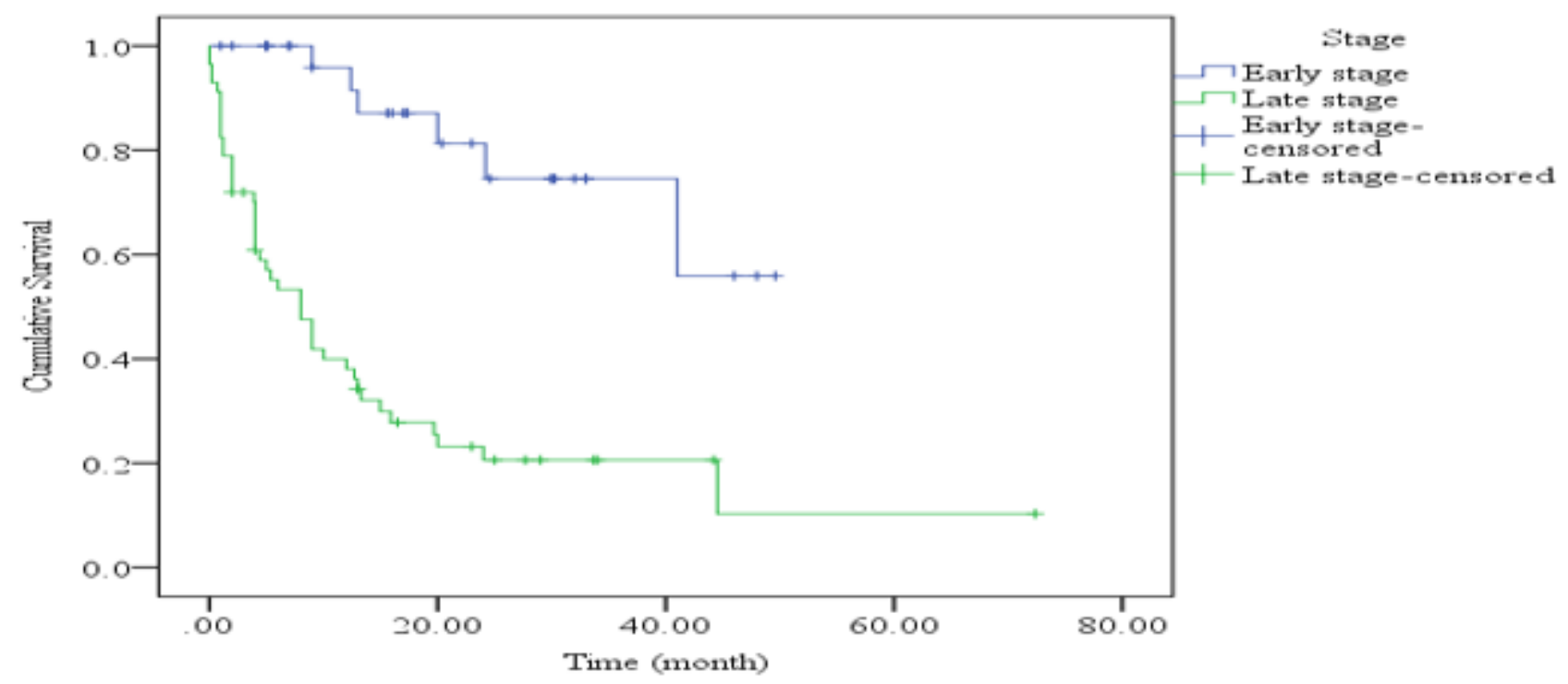

Figure 3

overall survival estimates of STS in relation to the disease stage 
Figure 4: Overall Survival estimates of RMS in relation to the histologic subtypes

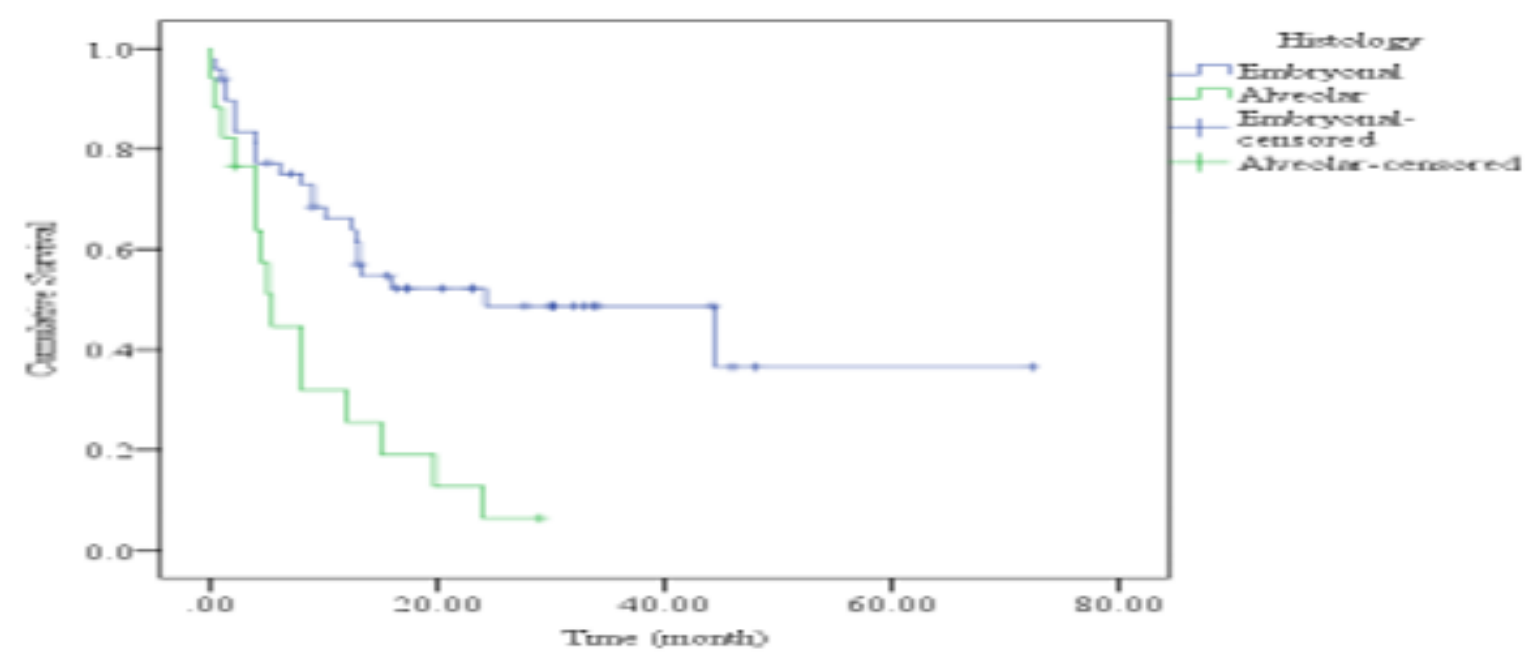

Figure 4

overall survival estimates of RMS in relation to the histologic subtypes

Figure 5: Overall Survival rate of bone sarcomas

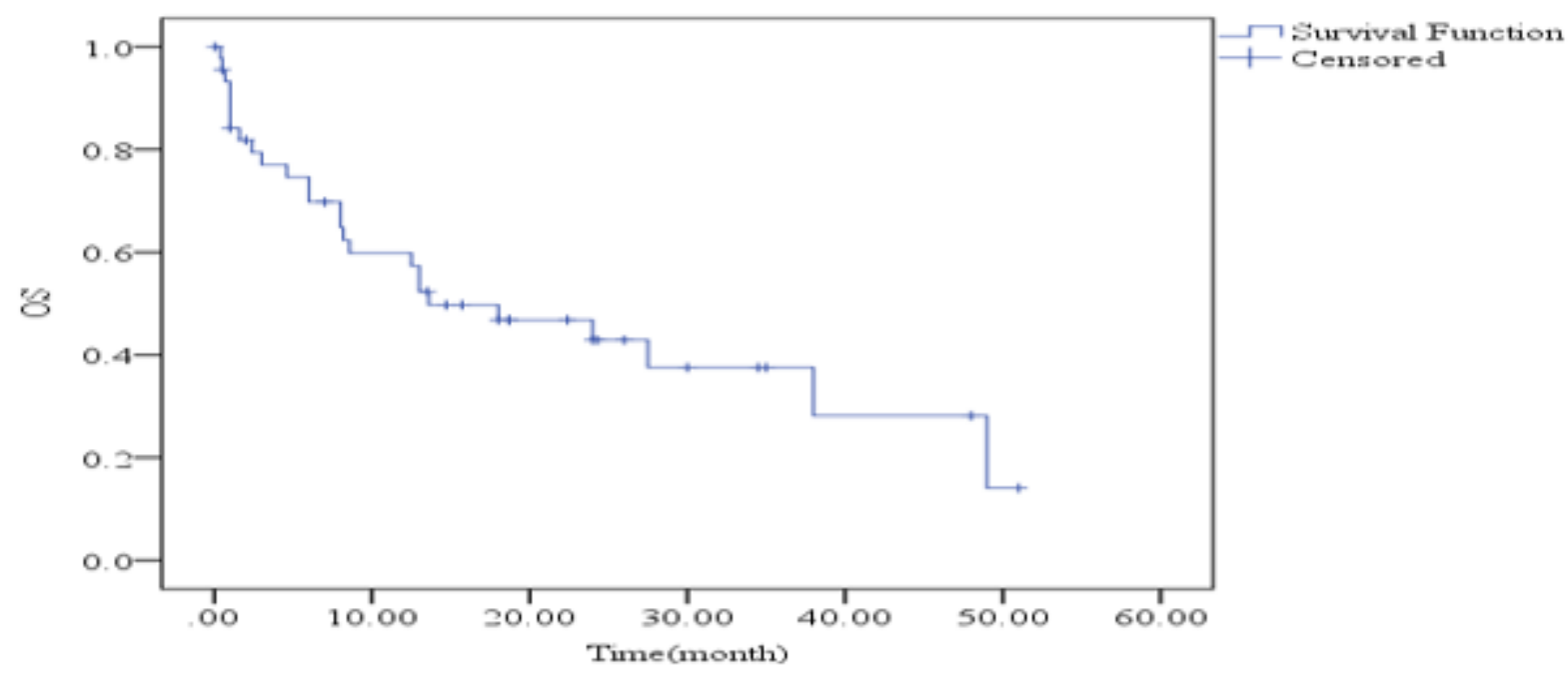

Figure 5

overall survival rate of bone sarcomas 
Figure 6: Overall Survival of bone sarcomas in relation to the disease stage

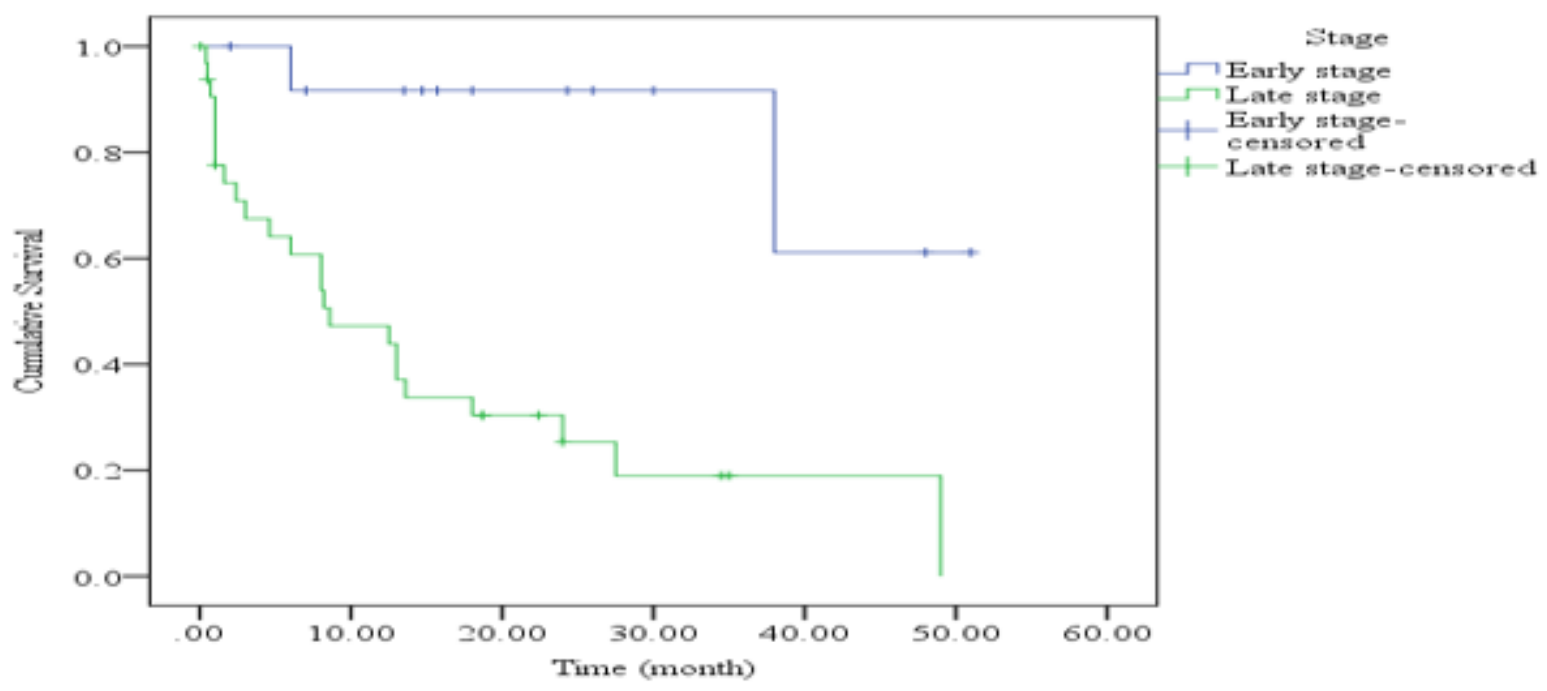

Figure 6

overall survival rate of bone sarcomas in relation to the disease stage

\section{Supplementary Files}

This is a list of supplementary files associated with this preprint. Click to download.

- supplement1.pdf 